\title{
Efficiency of Rapid Antigen Test in Diagnosis of Acute Streptococcal Tonsillopharyngitis
}

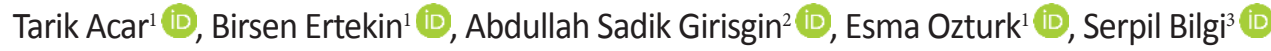 \\ ${ }^{1}$ Beyhekim State Hospital, Emergency Department, Konya, Turkey. \\ ${ }^{2}$ Necmettin Erbakan University, Meram Faculty of Medicine, Department of Emergency, Konya, Turkey. \\ ${ }^{3}$ Beyhekim State Hospital, Microbiology Department, Konya, Turkey. \\ Correspondence Author: Tarik Acar \\ E-mail: drtarikacar@gmail.com \\ Received: 18.04.2018 Accepted: 27.07.2018
}

\begin{abstract}
Objective: Group A beta hemolytic streptococcus (GABHS) is the most common bacterial cause of acute tonsillopharyngitis. Cases with bacterial infection suspicion should undergo rapid antigen test (RAT) and/or throat culture test in addition to clinical criteria, since it may lead to serious complications.

Method: A total of 220 adult and pediatric patients admitted to the emergency department between April-May 2016 with complaints of fever and sore throat, and diagnosed as acute tonsillopharyngitis were prospectively enrolled to the study. All participants had Centor score $\geq 2$ and they underwent RAT. Sensitivity, specificity, positive predictive value (PPV) and negative predictive value (NPV) of RAT results of both groups were compared. Throat culture was considered as gold standard method.

Results: Mean age of the study population was $22,5 \pm 16,9(1-72)$ years. $57 \%(n=61)$ of the pediatric group, and $42.5 \%$ ( $n=48)$ of adult group were male. Sensitivity of RAT in adults and pediatric group was $62 \%$ vs. $57 \%$, and specificity of the test was $96 \%$ vs. $97 \%$, PPV was $55 \%$ vs. $80 \%$, and NPV was $97 \%$ vs. $93 \%$, respectively.

Conclusion: We found low sensitivity and PPV values of RAT. Also, low levels of sensitivity decrease the possibility of RAT to be a diagnostic tool for the diagnosis of GABHS.

Keywords: Pharyngitis, streptococci, emergency care, rapid antigen test, culture
\end{abstract}

\section{INTRODUCTION}

Acute tonsillopharyngitis is a benign and self-limiting disease characterized with sore throat and difficulty in swallowing. It is the cause of $1-2 \%$ of total outpatient clinic administrations in United States which makes 12 million patients (1). These applications cause needless antibiotic prescriptions (2). Most of tonsillopharyngitis cases are viral in origin however; bacterial etiology must be excluded because of its serious complications (3). The most common cause of bacterial tonsillopharyngitis is Group A beta hemolytic Streptococcus (GABHS). Certain diagnosis and rapid onset of antibiotherapy is crucial since it may lead to serious complications such as acute rheumatic fever and acute glomerulonephritis (4).

Clinicians should consider rapid antigen test (RAT) and/or throat culture test in addition to clinical diagnostic criteria in patients with bacterial infection suspicion while establishing streptococcal tonsillopharyngitis diagnosis (5). Thus, a number of scoring system criteria were developed to help identifying bacterial agent. Modified Centor Criteria is most commonly used one. Throat culture positivity rate is $56 \%$ in patients with 4 criteria, whereas that probability is $2.5 \%$ in patients with 0 criteria for the diagnosis of GABHS (6).
But sensitivity (12\%-92\%) and specificity (30\%-93\%) of this criterion are reported variously (7).

Although culture is the gold standard diagnostic test for streptococcal pharyngitis this takes 2 days for the isolation of the microorganism. Sensitivity of culture in GABHS identification is reported as $90-95 \%$ (8). Nevertheless, laboratory requirements, cost, and duration of throat culture limits its use as a diagnostic tool in low-income or developing countries. On the other hand, RAT is a diagnostic test with high sensitivity and specificity which gives result within minutes (9). Rapid antigen test enables early initiation of treatment and subsequently symptomatic relief within 48 hours. Furthermore, this precludes spreading of the microorganism and needless prescription of antibiotics (10).

Rapid antigen test has been used in Europe and United States for a long time; however, it is not commonly used in our country, especially in the emergency departments yet. Thus, in this study we aimed to investigate diagnostic value of RAT for GABHS on patients admitted to emergency department with acute tonsillopharyngitis according to Centor classification, its relation with throat culture results, and its effect on antibiotic prescription rate. 


\section{METHODS}

A total of 220 adult and pediatric patients admitted to the emergency department of a state hospital with fever and sore throat complaints between April-May 2016 and diagnosed with acute tonsillopharyngitis were prospectively enrolled to the study. Demographic data, complaints, physical examination findings and vital signs of the participants were recorded. All patients applying Centor criteria which includes $38 \geq$ fever by history, tonsillar exudates, tender anterior cervical adenopathy, age and absence of cough, acute rhinorrhea were divided into two: above (adults) and below (pediatric) 18 years of age. All participants had $\geq 2$ score according to Centor classification. Exclusion criteria were as follows: antibiotic usage in last 2 weeks, patients with a history of acute rheumatic fever or acute glomerulonephritis. Patients with Centor score 0-1 were discharged after symptomatic treatment and recommendations. Rapid antigen test was applied to the ones with Centor score $\geq 2$. Two study groups (adults vs. pediatric group) were compared in terms of sensitivity, specificity, PPV and NPV of their RAT results. Throat culture test was accepted as gold standard method. Furthermore, the clinicians were asked about their antibiotic prescription decision after physical examination before they noticed the RAT results. Both groups were compared in terms of RAT results and the clinician's decision on antibiotic prescription. The present study was compatible with ethical rules of Helsinki Declaration. All participants or their parents signed informed consent form. Local ethics committee of Necmettin Erbakan University, Meram Faculty of Medicine approved the study protocol (date: 2017, number: 872).

\subsection{Microbiologic Method}

Two throat swabs were carefully (avoiding to touch tongue, uvula, or elsewhere) collected from oropharynx of each patient by an experienced doctor who evaluated and examined the patient. The first collected swab was exposed to RAT which is an immunochromatographic test used to calitatively detect StrepA antigen (Turklab Strep A Test/ İzmir/Turkey). Second swabs were taken into Stuart agar which contains sheep blood (BioMerieux/Istanbul/Turkey) seeded and then incubated for 24 hours. Catalase test was performed on the reproducing and beta hemolysis making colonies while culture plates were evaluated. Catalasenegative ones were exposed to PYR (L-pyronidonin beta naphthylamide). PYR (+) colonies were taken into passage and loaded into automated antibiogram and identification device (VItek ${ }^{\circledR}$ 2; Healthcare/bioMerieux/USA). The bacterial strains identified as Streptococcus pyogenes were reported as 'GABHS (Strep. pyogenes) reproduction'. Other identified strains were reported as 'normal oral flora reproduction' (11, 12).

\subsection{PYR Method}

PYR-impregnated papers were immersed to distilled water and inseminated with beta hemolysis catalase-negative bacteria which degrade L-pyronidonine beta naphthylamide by the naphthylamidase enzyme. Then indicator is added 2 minutes later. Pink color indicated the presence of PYR. Enterococci, S.pyogenes and Staphylococcus lugdunensis are also PYR-positive. Thus, if the detected colonies are catalasenegative and PYR-positive, they are identified as S. pyogenes by the automated bacteria identification (13).

\subsection{Statistical Analysis}

All data was analyzed with SPSS 21.0 software (IBM Turk Limited Company /Istanbul/Turkey). All variables were summarized by descriptive variables. Kolmogorov-Smirnov test was used to verify that continuous variables were normally distributed. Continuous variables were listed as mean \pm standard deviation, and categorical variables were listed as percentages (\%). Chi-square test was used to compare categorical variables of independent groups. McNemar test was used for comparison of categorical variables of 2 dependent groups. Sensitivity, specificity, PPV, and NPV of RAT results of both groups were calculated. Statistical significance was defined as $p \leq 0.05$.

\section{RESULTS}

Mean age (minimum-maximum) of the study population $(n=220)$ was $22.5 \pm 16.9(1-72)$ years. Mean age of the pediatric group ( $n=107)$ was $7.9 \pm 4.3$ years, and that of adult group $(n=113)$ was $36.3 \pm 12.2$ years. Fifty-seven percent $(n=61)$ of the pediatric group, and $42.5 \%(n=48)$ of the adults were male. The antibiotic prescription rate of the clinicians who had examined the patient and unannounced about the RAT (-) and throat culture (-) results of the patient was $54.2 \%(n=58)$ in pediatric group, and $43.3 \%(n=49)$ among adult group (107 of 220 (48.6\%) patients in total). Pediatric and adult groups were comparable in terms of RAT results $(p=0.450)$ and throat culture results $(p=0.104)$. There was a statistically significant difference between study groups in antibiotic prescription decision rates on the basis of clinical findings ( $p=0.005$ ) (Table 1 and 2). Rapid antigen test sensitivity was $62 \%$ vs. $57 \%$, specificity was $96 \%$ vs. $97 \%$, PPV was $55 \%$ vs. $80 \%$, and NPV was $97 \%$ vs. $93 \%$ in adult vs. pediatric group, respectively. Table 3 and 4 demonstrates false-positive and false-negative RAT results of RAT in adult and pediatric groups, respectively.

Table 1. Decision to prescribe antibiotics in childhood

\begin{tabular}{|l|l|c|c|c|}
\hline \multirow{2}{*}{} & & \multicolumn{2}{|c|}{ Culture } & Total \\
\hline \multirow{2}{*}{$\begin{array}{l}\text { Decision of } \\
\text { doctors }\end{array}$} & Antibiotic Writing & 14 & 61 & 75 \\
\cline { 2 - 5 } & Antibiotic isn't Writing & 0 & 32 & 32 \\
\hline Total & & 14 & 93 & 107 \\
\hline
\end{tabular}

Sensitivity:100\%; Specificity:34\%; PPV:18\%; NPV:100\% ; Consistency: 42\% 
Table 2. Decision to prescribe antibiotics in adults

\begin{tabular}{|l|l|c|c|c|}
\hline \multirow{2}{*}{} & & \multicolumn{2}{|c|}{ Culture } & Total \\
\hline \multirow{2}{*}{$\begin{array}{l}\text { Decision of } \\
\text { doctors }\end{array}$} & Antibiotic Writing & 8 & 51 & 59 \\
\cline { 2 - 5 } & Antibiotic isn't Writing & 0 & 54 & 54 \\
\hline Total & & 8 & 105 & 113 \\
\hline & & & & \\
\hline
\end{tabular}

Sensitivity:88\%; Specificity:51\%; PPV:13\%; NPV:100\% ; Consistency: 54\%

Table 3. RAT results in childhood

\begin{tabular}{|l|c|c|c|c|}
\hline & & \multicolumn{2}{|c|}{ Culture } & Total \\
\hline \multirow{3}{*}{ RAT } & & Positive & Negative & \\
\cline { 2 - 5 } & Positive (+) & $\begin{array}{c}\text { True }(+) \\
8\end{array}$ & $\begin{array}{c}\text { False }(+) \\
2\end{array}$ & 10 \\
\cline { 2 - 5 } & Negative (-) & $\begin{array}{c}\text { False }(-) \\
6\end{array}$ & $\begin{array}{c}\text { True }(-) \\
91\end{array}$ & 97 \\
\hline Total & & 14 & 93 & 107 \\
\hline
\end{tabular}

Sensitivity:57\%; Specificity:97\%; PPV:80\%; NPV:93\% ; Consistency: $90 \%$ RAT:Rapid Antigen Test

Table 4. RAT results in adults

\begin{tabular}{|c|c|c|c|c|}
\hline & & \multicolumn{2}{|c|}{ Culture } & Total \\
\hline & & Positive & Negative & \\
\hline \multirow{2}{*}{ RAT } & Positive (+) & $\begin{array}{c}\text { True }(+) \\
5\end{array}$ & $\begin{array}{c}\text { False }(+) \\
4\end{array}$ & 9 \\
\hline & Negative (-) & $\begin{array}{c}\text { False }(-) \\
3 \\
\end{array}$ & $\begin{array}{c}\text { True (-) } \\
101 \\
\end{array}$ & 104 \\
\hline TOTAL & & 8 & 105 & 113 \\
\hline
\end{tabular}

Sensitivity:62\%; Specificity:96\%; PPV:55\%; NPV:97\% ; Consistency: 93\% RAT:Rapid Antigen Test

\section{DISCUSSION}

We found RAT sensitivity as $62-57 \%$, specificity as $96-97 \%$, PPV as $55-80 \%$, and NPV as $97-93 \%$ when we applied RAT and throat culture in adult and pediatric patients having Centor score $\geq 2$ in our study. When we compared the HAT and culture outcomes of adult and pediatric patients, the results were clinically similar. Besides, our results regarding test sensitivity and PPV values were much lower than those specified by the manufacturer (sensitivity: $97.3 \%$, specificity: 99\%, PPV: 98.6\% and NPV: 97.5\%).

A rapid, easy and readily available method using throat swab to detect streptococci, RAT, is being used as a diagnostic tool in recent years. This test has advantageous of giving result within minutes during diagnostic study and has a sensitivity of $70-80 \%$ and a specificity of $95 \%$ when used with the gold standard throat culture test. However, RAT sensitivity may differ between $75 \%$ to $95 \%$ according to the kit and the study design (14). This variation was thought to arise from study groups, culture methods, laboratory performance and difference in disease spectrum. One of the most important factors affecting test sensitivity is gathering throat swab correctly. The American Academy of Pediatrics (AAP) guideline reports that RAT sensitivity differs between $70 \%$ 95\% according to the swab gathering technique and staff experience (15). Besides, colony amount on the swab may affect RAT sensitivity. A number of recent study emphasize that RAT sensitivity varies according to disease severity (16, 17).

When we consider variation in RAT sensitivity, IDSA, AAP, the American Heart Association (AHA) and other recent guidelines recommend verifying negative RAT results with throat culture which has higher sensitivity (18). Studies evaluating RAT and throat culture report RAT sensitivity as 87-96.7\%, RAT specificity as $95-100 \%$, PPV as $84.5-95 \%$, and NPV as $95.1-100 \%(9,19)$. Gurol et al. found RAT sensitivity as $64.6 \%$ which is much lower than the value $(95 \%)$ declared by manufacturer, but higher than the value (58\%) reported in literature (58\%) (20). Çamurdan et al. reported RAT sensitivity as $97.2 \%$ in their study and they state that this difference may be originating from the trademark of the kit they used (21). A study by Cardoso et al. demonstrated that PPV of RAT is $44.9 \%$ and $32.9 \%$ of the cases were prescribed with unnecessary antibiotic (11) The most probable explanation of this low sensitivity in our study depends on various factors that affect RAT sensitivity such as swab collecting technique, variety in staff experience, throat flora of patient at that moment, and inadequate time allowed to each patient in emergency departments, as mentioned in the literature.

Araujo et al. detected a false-positive rate of $32.6 \% \quad(n=15)$ and speculated that this had arisen from improper technique of rapid test they used or from cross-reaction of other groups with streptococci. Also, they pointed out that usage of eau for oral hygiene prevents microorganisms to reproduce in culture media properly (22). We found false-positive result in only $3.5 \%(n=4)$ of adult group and in $1.8 \%(n=2)$ of pediatric group. On the other hand, our study demonstrated that if we didn't administer RAT; clinicians would decide to prescribe unnecessary antibiotics to $43.3 \%(n=49)$ of adults and $54.2 \%(n=58)$ of pediatric patients according to clinical findings. Consequently, usage RAT precludes prescription of unnecessary antibiotics especially in pediatric patients.

Mayes et al. point out the fact that if false-negative rate of RAT decrease below $2.4 \%$, it can replace throat culture (23). According to Infectious Diseases Society of America (IDSA), because streptococcal pharyngitis and acute rheumatic fever are very rare for children $<3$ years old, there is no need for further diagnostic studies in this age group except for selected children in GABHS pharyngitis (24). But Çamurdan et al. reported false-negative rate of RAT as $6.1 \%$ which is lower than that of many previous studies, and stated that negative test results should be verified with throat culture in countries where GABHS infection complications are frequently encountered such as Turkey (21). The study by Araujo Filho et al. reported NPV of RAT as $94.2 \%$ and $6 \%$ $(n=2)$ false-negative result. They speculated that this might originate from restricted amount of antigens located in oropharynx (22). Darrow et al. proposed the explanation of RAT negativity in culture $(+)$ patients as insufficient amount of swab material collection during RAT procedure, besides rarely presence of scarce amount of colonies in culture 
media (25). We found false-negative RAT result rate only $2.6 \%(n=3)$ among adults and $5.7 \%(n=6)$ in pediatric group. Thus, only 9 in 220 patients (4\%) did not receive antibiotic treatment although they needed. Throat culture results were followed-up and patients with positive culture results were recalled. Hence, it is important to keep in mind that RAT results should be re-evaluated concomitantly with culture results especially in countries where RAT sensitivity is low and affected by many factors, and GABHS infection and its complications are frequently encountered like our country.

There is no international consensus currently on RAT usage in streptococcal tonsillopharyngitis diagnosis. Nonetheless, these kits are being commonly used in Europe and United States (26). There are a number of advantages of RAT though variations in its sensitivity rates. Application of this test in laboratory, clinics and emergency departments is easy which give result in less than 15 minutes. In this way, RAT provides rapid and reliable diagnosis of GABHS and decrease nonsuppurative complications of the infection and precludes unnecessary antibiotic prescription rates (20). Maltezou et al. reported $61 \%$ decrease in unnecessary antibiotic prescription rate with RAT use (12). Improper antibiotic usage leads to drug side effects, antibiotic resistance development, and consequent increase in health service cost. Rapid antigen test can provide economic gain in health expenditures if its sensitivity is high enough (27).

Limitations of this study;

1- The cost of throat culture test performed at emergency departments is not included in health insurance in our country. Also, RAT cost is relatively high which limits patient number included into study.

2- The factors that affect oral flora (such as oral hygiene, smoking, use of eau for oral hygiene, etc.) were not evaluated during throat swab collection which was done and evaluated at emergency department by unstandardized various health staff. This might influence test results.

3- The participants had not been followed-up in terms of drug side effects, antibiotic resistance, or hospital re-admission. Thus, we could not make an extensive calculation of cost effectiveness.

\section{CONCLUSION}

We found lower sensitivity and PPV of RAT in patients admitted with acute tonsillopharyngitis than those declared by the manufacturer and by previous studies in the literature. Whereas, our RAT specificity and NPV of RAT were higher than those reported before. Thus, low levels of sensitivity decrease the possibility of RAT to be a diagnostic tool for the diagnosis of GABHS. But all factors affecting RAT sensitivity should be kept in mind. Additionally, low false-positivity rate of RAT decreases unnecessary antibiotic prescription tendency of doctors at emergency department who decide according to clinical criteria only. Further extensive studies with higher patient population are needed to clarify the benefits of RAT use in GABHS diagnosis.

\section{REFERENCES}

[1] Schappert SM, Rechtsteiner EA. Ambulatory medical care utilization estimates for 2006. Natl Health Stat Report 2008; 8: 1-29.

[2] Barnett ML, Linder JA. Antibiotic prescribing to adults with sore throat in the United States, 1997-2010. JAMA Intern Med 2014;174(1):138-140.

[3] Alcaide ML, Bisno AL. Pharyngitis and epiglottitis. Infect Dis Clin North Am 2007;21(2):449-469.

[4] Spellerberg B, Brandt C. Streptococcus. Murray PR, Baron EJ, Jorgensen JH, Landry ML, Pfaller MA editors. Manual of clinical microbiology. ASM Press, Washington D.C.; 2007: 412-429.

[5] Harris AM, Hicks LA, Qaseem A. Appropriate antibiotic use for acute respiratory tract infection in adults. Ann Intern Med 2016;165(9):674.

[6] Centor RM, Witherspoon JM, Dalton HP, Brody CE, Link K. The diagnosis of strep throat in adults in the emergency room. Med Decis Making 1981;1(3):239-246

[7] Regoli M, Chiappini E, Bonsignori F, Galli L, de Martino M. Update on the management of acute pharyngitis in children. Ital J Pediatr 2011;37:10.

[8] Bisno AL, Gerber MA, Gwaltney Jr JM, Kaplan EL, Schwartz RH. Practice guidelines for the diagnosis and management of group A streptococcal pharyngitis. Clin Infect Dis 2002;35(2):113125.

[9] Fontes MJ, Bottrel FB, Fonseca MT, Lasmar LB, Diamante R, Camargos PA. Early diagnosis of streptococcal pharyngotonsillitis: assessment by latex particle agglutination test. J Pediatr (Rio J) 2007;83(5):465-470.

[10] Sheeler RD, Little P. Rapid streptococcal testing for sore throat and antibiotic resistance. Clin Microbiol Infect 2006; 12 (9): 3-7.

[11] Cardoso DM, Gilio AE, Hsin SH, Machado BM, de Paulis M, Lotufo JP, Martinez MB, Grisi SJ.Impact of the rapid antigen detection test in diagnosis and treatment of acute pharyngotonsillitis in a pediatric emergency room.Rev Paul Pediatr 2013;31(1):4-9.

[12] Maltezou HC, Tsagris V, Antoniadou A, Galani L, Douros C, Katsarolis I. Evaluation of a rapid antigen detection test in the diagnosis of streptococcal pharyngitis in children and its impact on antibiotic prescription. J Antimicrob Chemother 2008;62(6):1407-1412.

[13] York MK, Traylor MM, Hardy J, Henry M. Biochemical tests for the identification of aerobic bacteria. PYR (L-Pyrrolidonylß-Naphthylamide) Test. In: Garcia LS (editor in chief). Clinical Microbiology Procedures Handbook. 3rd ed. update, ASM Press, Washington D.C. 2010, p. 3.17.41.1 - 3.17.41.3

[14] Gerber MA, Shulman ST. Rapid diagnosis of pharyngitis caused by group A streptococci. Clin Microbiol Rev 2004; 17(3): 571580.

[15] Pickering LK. 2000 Red Book: Report of Committee on Infectious Diseases. 25nd ed. Elk GroveVillage; 2000.

[16] Tanz RR, Gerber MA, Kabat W, Rippe J, Seshadri R, Shulman ST. Performance of a rapid antigen-detection test and throat culture in community pediatric offices:implications for management of pharyngitis. Pediatrics 2009;123(2):437-444. 
[17] Dimatteo LA, Lowenstein SR, Brimhall B, Reiquam W, Gonzales $R$. The relationship between the clinical features of pharyngitis and the sensitivity of a rapid antigen test: evidence of spectrum bias. Ann Emerg Med 2001;38(6):648-652.

[18] Rimoin A.W, Walker CL, Hamza HS, Elminawi N, Ghafar HA, Vince $A$. The utility of rapid antigen detection testing for the diagnosis of streptococcal pharyngitis in low-resource settings. Int J Infect Dis 2010;14(12):1048-1053.

[19] Santos AG, Berezin EN. Comparative analysis of clinical and laboratory methods for diagnosing streptococcal sore throat. J Pediatr (Rio J) 2005;81(1):23-28.

[20] Gurol Y, Akan H, Izbirak G, Tekkanat ZT, Gunduz TS, Hayran $O$. The sensitivity and the specifity of rapid antigen test in streptococcal upper respiratory tract infections. Int J Pediatr Otorhinolaryngol 2010;74(6):591-593.

[21] Camurdan AD, Camurdan OM, Ok I, Sahin F, Ilhan MN, Beyazova U. Diagnostic value of rapid antigen detection test for streptococcal pharyngitis in a pediatric population. Int J Pediatr Otorhinolaryngol 2008;72(8):1203-1206.

[22] Araujo Filho BC, Imamura R, Sennes LU, Sakae FA. Role of rapid antigen detection test for the diagnosis of
group-A beta-hemolytic streptococcus in patients with pharyngotonsillitis.Braz J Otorhinolaryngol 2006;72(1):12-15.

[23] Mayes TM, Pichichero ME. Are follow-up throat cultures necessary when rapid antigen detection tests are negative for group A streptococci? Clin Pediatr (Phila) 2001;40(4):191-195.

[24] Shulman ST, Bisno AL, Clegg HW, Gerber MA, Kaplan EL, Lee $G$. Clinical practice guideline for the diagnosis and management of group A streptococcal pharyngitis: 2012 update by the infectious diseases society of America. Clin Infect Dis 2012;55(10):1279-1282.

[25] Darrow DH, Siemens C. Indications for tonsillectomy and adenoidectomy. Laryngoscope. 2002;112(100): 6-10.

[26] Matthys J, De Meyere M, van Driel ML, De Sutter A. Differences among international pharyngitis guidelines: not just academic. Ann Fam Med 2007;5(5):436-443.

[27] Rimoin AW, Walker CL, Hamza HS, Elminawi N, Ghafar HA, Vince $A$. The utility of rapid antigen detection testing for the diagnosis of streptococcal pharyngitis in low-resource settings. Int J Infect Dis. 2010;14(12):1048-1053. 